\section{Dr. Kang, et al, reply}

To the Editor:

We were surprised to read the letter by Berti and colleagues ${ }^{1}$, commenting on our recent article on the incidence of arterial and venous thrombosis in antineutrophil cytoplasmic antibody-associated vasculitis (AAV) ${ }^{2}$. They make the unjustified comment that "the incidence estimates for [arterial (ATE) and venous thrombosis events (VTE)] may be inflated" when we give clear and accurate incidence rates. Our incidence of ATE was 2.67/100 patient-years (PY), whereas the 2018 article by Berti, et $a l^{3}$ reports an incidence of cardiovascular disease as 5.0/100 PY. It would therefore not seem that our incidence estimates were inflated.

Our comparison of the characteristics of patients who did or did not develop ATE or VTE is entirely valid. Berti, et $a l^{1}$ make the point that a history of previous ATE predicts the risk of subsequent ATE. We discuss this possibility in our article, and that is why we performed the sensitivity analysis, which indeed shows a lower risk of ATE in those without a history of previous ATE. This was consistent with our regression analysis. After adjusting for other covariates, previous ischemic heart disease remains a significant risk factor for subsequent ATE. We acknowledge that the Kaplan-Meier plots are not the ideal way in which to compare survival between the 2 groups with and without thrombotic events. However, our median followup time was greater than 5 years, and the majority of thrombotic events occurred early during the disease course. The Kaplan-Meier plots provide a graphical representation of outcome (survival) limited to the followup time concerned.

Regarding Berti and colleagues' point about comparing thrombotic events in AAV to crude rates in the UK population, we agree that this is not an ideal comparison, but it was not practical for us to identify appropriate matched controls. Few places have access to the population data available in Omsted County (USA). We clearly state that we are comparing our results with those of the general population and acknowledge this is not an ideal approach. We also acknowledge as a limitation the fact that we could not obtain information on all the risk factors for thrombotic events in this retrospective study. In addition, we compare our incidence rates of ATE and VTE with published literature for AAV and other inflammatory diseases. Our results seem similar to several of these previous studies and generally show a higher rate of events in the first year after diagnosis, possibly related to disease activity.

Berti, et $\mathrm{ll}^{1}$ seem concerned about our statement that we were the first to report a high incidence of ischemic stroke in AAV. They reference their article published in May 2018 ${ }^{3}$. As experienced investigators, they should surely realize that our revised manuscript was submitted to The Journal of Rheumatology well before their publication was released. If their publication had been available, we would of course have quoted it, as we did others in the literature.
Overall, we are disappointed by the critical tone taken by Berti, et al ${ }^{1}$, as we were doing our best, in a single-center retrospective study, to draw to the reader's attention the high incidence of ATE and VTE in patients with AAV. Indeed, their own paper reports broadly similar results and supports our conclusion.

AMY KANG @ , FRACP, MBBS (Hons), Clinical Fellow, Imperial College Renal and Transplant Centre, Imperial College Healthcare National Health Service (NHS) Trust; MARILINA ANTONELOU (1), MRCP, BSc, Academic Clinical Fellow, Imperial College Renal and Transplant Centre, Imperial College Healthcare NHS Trust; NIKKI L. WONG, FRACP, MBBS, Clinical Research Fellow, Imperial College Renal and Transplant Centre, Imperial College Healthcare NHS Trust; ANISHA TANNA, MBBS, BSc, Clinical Research Training Fellow, Renal and Vascular Inflammation Section, Department of Medicine, Imperial College London; NISHKANTHA ARULKUMARAN ${ }^{\circ}$, PhD, MBBS, Renal Specialty Registrar, Imperial College Renal and Transplant Centre, Imperial College Healthcare NHS Trust; FREDERICK W.K. TAM ${ }^{0}$, PhD, MB BChir, Ken and Mary Minton Chair of Renal Medicine, Renal and Vascular Inflammation Section, Department of Medicine, Imperial College London; CHARLES D. PUSEY (1), DSc, MB BChir, Professor of Medicine, Renal and Vascular Inflammation Section, Department of Medicine, Imperial College London, London, UK. Address correspondence to C.D. Pusey, Renal and Vascular Inflammation Section, Department of Medicine, Imperial College London, Hammersmith Campus, Du Cane Road, London W12 0NN, UK. E-mail: c.pusey@imperial.ac.uk

\section{REFERENCES}

1. Berti A, Matteson EL, Crowson CS, Specks U, Cornec D. The incidence of arterial and venous thrombosis in antineutrophil cytoplasmic antibody-associated vasculitis. J Rheumatol 2019;46:1243.

2. Kang A, Antonelou M, Wong NL, Tanna A, Arulkumaran N, Tam FW, et al. High incidence of arterial and venous thrombosis in antineutrophil cytoplasmic antibody-associated vasculitis. J Rheumatol 2019;46:285-93.

3. Berti A, Matteson EL, Crowson CS, Specks U, Cornec D. Risk of cardiovascular disease and venous thromboembolism among patients with incident ANCA-associated vasculitis: a 20-year population-based cohort study. Mayo Clin Proc 2018;93:597-606.

J Rheumatol First Release July 1 2019; 2019;46:9; doi: $10.3899 /$ jrheum. 181446 